16 MEDICAL STUDENTS ASSESSMENT OF EARLIER STUDYING AND PRACTICING CONCEPTS OF MEDICAL EDUCATION

1,2Mohammed Ahmed Abdelrahman, 'Tarig Sideeg, 'Marouf Mohammed. 'Faculty of Medicine and Health Sciences, Omdurman Islamic University, khartoum, Sudan; ${ }^{2}$ Educational Development Center-Sudan Medical Speacialization Board, khartoum, Sudan

\subsection{6/bmjebm-2019-EBMLive.97}

Objectives The aim of this work is to assess undergraduate medical student's opinions on the new trends in medical education course, and to test the effect of medical student's assessment of medical education course comparing male and female students.

Method Analytical cross-sectional institutional based study conducted at Faculty of Medicine (FMHS), Omdurman Islamic University (OIU), 2018. It was total coverage for all students of first level, therefore, 346 male and female medical students asked to fill pretested questionnaire that contains 9 questions of three domains of knowledge and skills was used to collect the data. The data collected were analyzed using (SPSS) version 20. Frequencies, percentage, Chi-squire were obtained to identify the correlations as analytical statistics between variables of the study. The confidence interval is $95 \%$ was taken as significant at $P$-value $\leq 0.05$.

Results There is no statically significant difference between male and female medical students regards assessment of trends in medical education course $(P$-value $>0.05)$, in spite they are taught separately. Both male and female students' assessment revealed positive responds with high percentage in cognitive knowledge, psychomotor and behavioral skills that studied in the course.

Conclusions Almost all students were ensured their agreement particularly in Self-learning, Small Group Work and Problem Based Learning (PBL) in the course.

In conclusion, no statistical difference between male and female medical students' assessment of trends in medical education course in this study.

\section{SCIENCE IS CONCEPTUALLY DYNAMIC AND CONSTANTLY TRYING TO RE-INVENTING ITSELF}

Augusto Cesar Soares dos Santos Junior 1,2,3. ${ }^{1}$ Unimed-BH, Belo Horizonte, Brazil; ${ }^{2}$ Hospital das Clinicas, Belo Horizonte, Brazil; ${ }^{3}$ Prefeitura Municipal, Contagem, Brazil

\subsection{6/bmjebm-2019-EBMLive.98}

Science is conceptually dynamic and constantly trying to reinventing itself. Since the beginning humankind has always been struggling on whether to go more general in its beliefs or to acknowledge diversity, allowing the construction of different solutions tailored to local particularities. Currently, it seems that researchers, patients, the pharmaceutical industry, governments, other stakeholders, are in midst of a debate where social, cultural and environmental characteristics have been historically overlooked as factors to be considered in the art of research and decision making. For decades we have built a framework where patients are being deliberately forced to fulfill a standard and reproduce the results of a study published elsewhere. Shouldn't it be the other way around? This failure to navigate between the search for universal truths and personalized healthcare has taken the patient from the center of the driving force of innovation. This dynamic allowed too much power in the hands of a few which have deliberately been trying to dictate the standards on how we behave and consume healthcare resources, opening the doors to incoherence, bias, conflicts of interests and frustration. Hopefully, in the near future, this search for truths, which forcibly must be applied to all, will be acknowledged as an oversimplified approach especially in the scenario of a world full of inequities and economic disparities. In this context, science is being called to shift its paradigms and acknowledge the complexity and diversity of humans. The overemphasized appeal of international publications, the irrational sophistication of the $\mathrm{p}<0.05$ statistics, the increasing importance of surrogate outcomes, the lack of transparency in research and the prohibitive costs of innovative treatments are just some of the hurdles which are leading communities around the globe to question current scientific practices. A feasible plan to be put into action is to bring research to the local level and acknowledge that humankind remains diverse in terms of expectations and experiences while challenged by illnesses or death. It's crucial to implement strategies fostering a broad education program in evidence-based medicine accompanied by funding disclosure from all involved parties and the creation of local real-world databanks. In addition, local talks, allowing all parties to clearly discuss the sustainability of the system and address issues like access and budget priorities must be put into the agenda. Taken together, these actions will clearly lead to a more balanced scenario, allowing more comprehensive local policies with less room to poor science. This new approach will hopefully be more sustainable, reduce the distance to good practices, increase bench-to-bedside research and correct the current framework which has historically neglected the importance of local background characteristics and beliefs. Acknowledging the diversity and allowing the construction of scientific solutions tailored to local particularities is the next wisest step to be further explored in the future developments of the evidence-based medicine movement. Unquestionably, this new approach will present a new set of challenges however it will also allow a new exciting outlook in the face of our current scientific practices.

\section{METHODOLOGY AND REPORTING QUALITY OF STUDIES USING MACHINE LEARNING MODELS FOR MEDICAL DIAGNOSIS: A METHODOLOGICAL SYSTEMATIC REVIEW}

${ }^{1}$ Mohamed Yusuf, ${ }^{2,3,4}$ Ignacio Atal, ${ }^{2,3}$ Jacques Li, ${ }^{5}$ Phil Smith, ${ }^{2,3,6}$ Philippe Ravaud, ${ }^{1}$ Michael Callaghan, 'James Selfe. 'Manchester Metropolitan University, Manchester, UK; ${ }^{2}$ INSERM, Methods, Université Paris Descartes, Paris, France; ${ }^{3}$ Cochrane France, Paris, France; ${ }^{4}$ Centre for Research and Interdisciplinarity (CRI), Université Paris Descartes, Paris, France; ${ }^{5}$ Manchester Metropolitan University, Manchester, France; ${ }^{6}$ EQUATOR-Network, Paris, France

\subsection{6/bmjebm-2019-EBMLive.99}

Objectives Over the past decade, due to access in large sums of clinical data and the development of new machine learning (ML) techniques, there has been a rise in the application of ML methods to medicine. Due to their propensity to facilitate and promote timely and objective clinical decision-making, ML methods have been applied to gain insights into clinical diagnosis. Despite their popularity, these promising techniques have pitfalls. Studies using $\mathrm{ML}$ for diagnosis may contain errors in both design and execution. Additionally, it appears ML researchers are not familiar with the agreed practices of the medical research community. 